\title{
A importância da avaliação da composição da qualidade do pirarucu durante o processamento pós colheita
}

The importance of evaluating the composition of the quality of pirarucu during post-harvest processing

\section{La importancia de evaluar la composición de la calidad del pirarucu durante el procesamiento}

poscosecha

\section{Resumo}

Introdução: o pirarucu é uma espécie nativo da amazônica, pertencente à ordem Osteoglossiformes, Arapaimidae. É um peixe com elevada importância econômica e um interesse comercial bastante crescente, devido suas características propícias para cultivo, tais como: a alta taxa de crescimento (até $10 \mathrm{~kg}$ no primeiro ano), elevada rusticidade ao manuseio, adaptabilidade à alimentação artificial e elevado aproveitamento da carcaça (51-57\%). Objetivo: O estudo parte de um levantamento bibliográfico acerca de artigos sobre alterações da qualidade microbiológica pós-colheita do pirarucu. Metodologia: Trata-se de uma pesquisa de revisão bibliográfica, descritiva. Resultado e Discussões: é indispensável o uso frequente de Boas Práticas de Manipulação, APPCC (Análise de Perigos e Pontos Críticos de Controle) para que haja sempre pescados com qualidade e seguros. É necessário manusear com cuidado a carne do pescado no processo de produção para não correr risco de contaminação. Estudos comprovam que a combinação da textura, aparência e sabor nos alimentos é fortemente essencial para a aceitação dos consumidores, para o controle de qualidade dos alimentos o conhecimento da composição dos alimentos no Brasil é fundamental. Conclusão: O pirarucu é um pescado de elevado valor cultural e comercial. O conhecimento dos fatores envolvidos (físicoquímicos, microbiológicos e sensoriais) na progressão da deterioração é fundamental para a segurança alimentar aos consumidores finais e para protocolos de beneficiamento e armazenamento ideal nas indústrias.

Palavras-chave: Pirarucu; Análise microbiológica; Composição centesimal.

\begin{abstract}
Introduction: The pirarucu is a species native to the Amazon, belonging to the order Osteoglossiformes, Arapaimidae. It is a fish with high economic importance and a very growing commercial interest, due to its favorable characteristics for cultivation, such as: high growth rate (up to $10 \mathrm{~kg}$ in the first year), high rusticity in handling, adaptability to artificial feeding and high utilization of the carcass (51-57\%). Objective: The study starts from a bibliographical survey about articles about alterations in the postharvest microbiological quality of pirarucu. Methodology: This is a descriptive bibliographic review. Results and Discussions: Frequent use of Good Handling Practices, HACCP (Hazard Analysis and Critical Control Points) is essential so that there are always quality and safe fish. Studies prove that the combination of texture, appearance and flavor in food is strongly essential for consumer acceptance, for the control of food quality, knowledge of the composition of food in Brazil is essential. It is necessary to handle the meat of the fish carefully in the production process so as not to run the risk of contamination. Conclusion: Pirarucu is a fish of high cultural and commercial value. Knowledge of the factors involved (physical-chemical, microbiological, sensory) in the progression of deterioration is essential for food security for end consumers and for processing and ideal storage protocols in industries.
\end{abstract}


Keywords: Pirarucu; Microbiological analysis; Proximate composition.

\section{Resumen}

Introducción: el pirarucu es una especie nativa del Amazonas, perteneciente al orden Osteoglossiformes, Arapaimidae. Es un pez de alta importancia económica y un interés comercial muy creciente, debido a sus características favorables para el cultivo, tales como: alta tasa de crecimiento (hasta $10 \mathrm{~kg}$ en el primer año), alta rusticidad en el manejo, adaptabilidad a la alimentación artificial y alta utilización de la canal (51-57\%). Objetivo: el estudio se basa en una encuesta bibliográfica sobre artículos sobre cambios en la calidad microbiológica poscosecha de pirarucu. Metodología: Se trata de una revisión bibliográfica descriptiva. Resultados y Discusiones: el uso frecuente de Buenas Prácticas de Manejo, HACCP (Análisis de Peligros y Puntos Críticos de Control) es fundamental para que siempre haya pescado de calidad y seguro. Es necesario manipular la carne del pescado con cuidado en el proceso de producción para no correr el riesgo de contaminación. Los estudios demuestran que la combinación de textura, apariencia y sabor en los alimentos es muy esencial para la aceptación del consumidor, para el control de la calidad de los alimentos, el conocimiento de la composición de los alimentos en Brasil es esencial. Conclusión: Pirarucu es un pez de alto valor cultural y comercial. El conocimiento de los factores involucrados (físico-químicos, microbiológicos y sensoriales) en la progresión del deterioro es fundamental para la seguridad alimentaria de los consumidores finales y para los protocolos de procesamiento y almacenamiento ideales en las industrias.

Palabras clave: Pirarucu; Análisis microbiológico; Composición centesimal.

\section{Introdução}

O pirarucu é uma espécie nativo da amazônica, pertencente à ordem Osteoglossiformes, Arapaimidae. E pode alcançar até $200 \mathrm{~kg}$ de peso total. É um peixe com elevada importância econômica e um interesse comercial bastante crescente, devido suas características propícias para cultivo, tais como: a alta taxa de crescimento (até $10 \mathrm{~kg}$ no primeiro ano), elevada rusticidade ao manuseio adaptabilidade à alimentação artificial e elevado aproveitamento da carcaça (51-57\%). O A. gigas é uma espécie de respiração aérea obrigatória, sendo que tal característica facilita sua criação em elevadas densidades de estocagem, bem como em baixas concentrações de oxigênio dissolvido na água (Crescêncio, 2001; Scorvo Filho, 2004).

Os fatores extrínsecos ou intrínsecos e a qualidade de seu frescor estão associados ao valor nutritivo do pescado, pois são relevantes para o processo de deterioração (Huss et al., 1998). Logo após a sua morte, o pescado passa por várias mudanças. O processo bioquímico, químico e físico é uma complexa combinação que pode ocasionar perda da qualidade do pirarucu. O peixe sofre perda da qualidade e frescor na matéria prima, causando odor desagradável, criando oportunidades para a proliferação bacteriana, isso pode ocorrer logo após sua estocagem e armazenamento com gelo, essa proliferação das bactérias levam o peixe ao acelerado processo de deterioração (Pacheco-Aguilar et al, 2000). O grau de conservação e o frescor do peixe são influenciados pelo método de captura, o tempo de arraste, áreas de pesca e refrigeração. Desde a sua captura até a comercialização, o pescado é passível de perda da qualidade (Soares \& Gonçalves, 2012).

Devido à grande atividade de água no pescado fica mais fácil a deterioração do peixe. Muitos fatores podem favorecer o desenvolvimento de microorganismos, como: a espécie, o teor de gordura, $\mathrm{pH}$, época do ano e sua composição química. (Landgraf, 1996).

$\mathrm{O}$ manejo do pescado pré-abate e o processamento durante o abate devem evitar o rigor mortis. $\mathrm{O}$ rigor mortis influência no produto final devido o endurecimento da carne do peixe. A duração do animal em rigor depende da quantidade de ATP e glicogênio do peixe (Robb \& Kestin, 2002).

Adicionalmente, as características sensoriais envolvem uma série de atributos com base em critérios de aparência, textura, odor, cor e sabor, sendo assim; para medir o processo de putrefação e sua intensidade, pode-se usar a composição centesimal, especialmente a concentração de proteína e lipídios. A conservação do pescado é determinada pelo pH, pois o processo de deterioração altera os níveis de $\mathrm{pH}$, devido à decomposição de aminoácidos, porém é indispensável outros parâmetros na determinação (Chytiri et al., 2004).

O método mais usual para avaliar os aspectos sensoriais da qualidade do pescado é o Método do Índice de Qualidade (MIQ), que deve ser desenvolvido para cada espécie de peixe. Vários estudos têm mostrado que, para peixes estocados em 
gelo, existe relação linear entre os escores do MIQ e o tempo de estocagem, o que possibilita a estimativa do tempo restante de vida útil do pescado (Baixas et al., 2003). É importante a realização de estudos sobre a composição química, sensorial e microbiológica devido a sua alta aceitação no mercado consumidor e o retorno socioeconômico que o pescado traz para sociedade.

O intervalo de tempo que o alimento pode ser conservado dependerá das condições de temperatura, umidade relativa, luminosidade e de oxigênio adequadas, de modo a preservar seus atributos sensoriais e nutricionais. Porém, continuam suscetíveis a ocorrer reações bioquímicas de deterioração (Tavares \& Gonçalves, 2011).

Visto que o pescado é propenso à deterioração devido suas características o objetivo do presente estudo parte de um levantamento bibliográfico acerca de artigos sobre alterações da qualidade microbiológica do pirarucu e entre outras espécies visando a melhoria da qualidade do pescado.

\section{Metodologia}

Trata-se de uma pesquisa bibliográfica na qual foi levantado os principais achados científicos na área, analisando-os e avaliando para contribuir no desenvolvimento do objetivo da pesquisa (KÖNCHE, 2011). Buscando correlacionar os estudos sobre a análise microbiológica, parâmetros físico-químicos e sensoriais que implicam na qualidade do pescado.

Uma revisão de literatura é um processo de busca, análise e descrição de uma série de conhecimentos com intenção de pesquisar a resposta a uma pergunta característica nas literaturas que são materiais completos sobre um tema sendo encontrados em: livros, artigos de periódicos, artigos de jornais, teses e dissertações entre outros (UNESP, 2015).Uma revisão de literatura é um processo de busca, análise e descrição de uma série de conhecimentos com intenção de pesquisar a resposta a uma pergunta característica nas literaturas que são materiais completos sobre um tema sendo encontrados em: livros, artigos de periódicos, artigos de jornais, teses e dissertações entre outros (UNESP, 2015).

Foram utilizadas as seguintes bases de dados: Google acadêmico, Scielo (Scientific Electronic Library Online), Repositório do INPA (Instituto Nacional de Pesquisa da Amazônia) e documentos oficiais.

Os materiais utilizados foram publicações gerais sobre o tema. Utilizando as seguintes palavras-chaves: pirarucu, análise sensorial do pescado, análise microbiológica pós abate, valor cultural do pirarucu, importância da composição nutricional dos alimentos e seus equivalentes em inglês.

Foram utilizados, estudos relacionados ao tema e os descritores de pesquisas: pirarucu, análise sensorial do pescado, análise microbiológica pós abate, valor cultural do pirarucu, importância da composição nutricional dos alimentos publicados com a prioridade dos últimos 10 anos (2011 - 2021).

Estudos anteriores aos anos de 2011, também foram incluídos por apresentarem relevância e reconhecimento sobre o tema, a justificativa para inclusão foi devida não haver publicações atuais sobre o assunto abordado.

Para seleção dos artigos incluídos foi realizada uma leitura prévia do resumo e a introdução, após a seleção inicial ocorreu a leitura completa do material e foi identificado os parágrafos de interesse.

\section{Resultados e Discussão}

\subsection{Arapaima gigas}

Desde os períodos de colonização a pesca é uma atividade de extrema importância na região Amazônica, sendo a pesca explorada pelos índios que utilizavam esses recursos para seu consumo devido já serem constituídos uma fonte protéica de qualidade para a manutenção humana. A quantidade de espécies de peixes na bacia amazônica é vasta, sendo considerado um número aproximado a 3 mil (Souza, 2016). Sendo o pirarucu (Arapaima gigas) uma dessas espécies amazônicas. 
O Arapaima gigas é o maior peixe amazônico com cerca de $200 \mathrm{~kg}$ e 3 metros, possui escamas grandes e formosas que são agradáveis aos artesãos. É um peixe amazônico, carnívoro, com cabeça grande e de forma achatada, e sua língua é óssea (Venturieri \& Bernardino, 1999; Gavis et al., 2006).

\subsection{Propriedades físicas}

Segundo Hylding e Nielsen (2001) quando o alimento é tocado e consumido, pode-se notar através da textura, sabor e aroma a avaliação sensorial espontânea do Arapaima gigas.

Estudos comprovam que a combinação da textura, aparência e sabor nos alimentos é fortemente essencial para a aceitação dos consumidores. Os métodos usados para avaliação e análise do alimento estão relacionados ao frescor detectado durante o consumo, sendo assim, para esse tipo de avaliação usa-se os métodos objetivos (Hylding \& Nielsen, 2001).

Primeiramente utiliza-se a avaliação através da mastigação, ou da textura pressionando com os dedos o alimento, esse método é avaliado por seres humano para adquirir unidade de conhecimento (conceito), o outro processo é avaliado através do texturômetro que é importantíssimo para a análise feitos por instrumentos científicos de extrema precisão da textura (Botta, 1991).

O estado de frescor tem influência intrínseco e extrínseca, é importante para avaliar a qualidade do peixe, textura e suas qualidades. Estudos de pesquisas elaboradas buscam correlacionar cor e textura com resultados sensoriais (Markore et al., 2002).

Trabalhos revisados por Hylding e Nielsen (2001) relatam que idade, tamanho e estado nutricional são fatores que influenciam na textura dos peixes, assim como o método usado para peixes inteiros e filés, são eles; glicose, pH, morte, temperatura, $\mathrm{NaCl}$ e estocagem que ocasiona modificação na textura após o abatimento. E ainda segundo a pesquisa dos autores as fibras dos peixes se tornam mais firmes e grossas decorrentes ao crescimento, sendo resultados positivos feitos em 7 espécies de peixe.

\subsection{Composição centesimal}

Para o controle de qualidade dos alimentos o conhecimento da composição dos alimentos no Brasil é fundamental, é importante também para a avaliação da ingestão de nutrientes de indivíduos ou populações, e ainda para atingir a segurança alimentar e nutricional no país. Esses dados podem direcionar a variação da alimentação, em oposição de uma dieta monótona e desequilibrada, e a produção agrícola e as indústrias de alimentos no processo de novos produtos. O objetivo da composição dos alimentos consiste em trazer informações atualizadas, confiáveis e adequadas (Torres et al., 2000; Taco, 2011).

O peixe é um dos alimentos mais apreciados e uma das principais fonte de alimento na Amazônia (Parente et al., 2005). O peixe é um alimento completo em questão nutricional, tanto na sua qualidade quanto sua macronutriente e micronutrientes (Córser et.al, 2000). A composição centesimal do pescado pode sofrer alteração por diversos motivos, sendo eles por questão intrínseca ou extrínseca. O pescado é de alto valor nutritivo, contendo 46 fontes de proteínas e diversas vitaminas. As proteínas têm alta digestibilidade ao redor de $90 \%$, sendo o coeficiente de eficiência superior ao da caseína $(2,9)$ (Kirschnik, 2007). Muitas literaturas apresentam diversos motivos que podem influenciar a composição centesimal do pescado, como idade, sexo, meio ambiente e estação do ano pois estão relacionadas com a alimentação, nado migratório e ciclo reprodutivo do pescado (Stansby, 1968; Contreras-Guzmán, 1994; Huss, 1998; Córser et al., 2000).

As proteínas do peixe são divididas em 3 grupos, de acordo com sua solubilidade: sarcoplasmáticas, miofibrilares e do estroma. Elas proporcionam viscosidade, gelatinização e textura nos alimentos. Tem alto teor de proteína (93\%) e os ácidos graxos do peixe são insaturados, sendo de bastante relevância nutritiva para os seres humanos (Oetterer, 2002). 
O principal componente do peixe é a água e sua parte comestível, varia entre $64 \%$ a $90 \%$, seguido pelas proteínas de $8 \%$ a $23 \%$ e pela gordura, de $0,5 \%$ a $25 \%$. Carboidratos não chegam a $1 \%$ da sua composição (Badolato, 1994). Stansby (1968) relata que de acordo com o teor de lipídios, os peixes podem ser classificados como: gordos (>15\%); semi-gordos (5 $15 \%)$ e magro (<5\%). Segundo Jacquot (1989), o teor de lipídio pode influenciar o teor de umidade no pescado. Dentre estas categorias tais percentuais de umidade variam entre $68,60 \%$ e $81,60 \%$. A composição química do peixe além de informar sobre aspectos nutricionais, proporciona conhecimento às indústrias de processamento, ajudam no controle de qualidade e nos métodos de preservação (Gurgel, 1972).

\subsection{Propriedades Microbiológica}

Para avaliar a qualidade microbiológica, química e sensorial é importante conhecer a forma de abate do peixe, o manejo após o sacrifício e o armazenamento (Ozogul \& Ozogul, 2004).

Segundo Centeno et al (2007), usa-se para determinação das características sanguíneas dos peixes os parâmetros metabólicos, Tavares Dias et al (2008) esse processo é importante para o manejo com peixes criados em represas, fornecem informações importantes para a saúde das pessoas.

O resultado das pesquisas realizadas através dos dados que envolvem estudos sobre o estado saudável dos peixes, estresse e enfermidades, correspondem a levantamentos sobre as morbidades da população (Tavares Dias \& Moraes, 2004).

Os trombócitos são estruturas presentes no sangue, fragmentos de citoplasma formados na medula óssea a partir das células, responsáveis pelo nível de estresse do peixe, também se relaciona com partículas sólidas e grandes (fagocitose) (Tavares-Dias et al., 2007).

A proteína do peixe é considerada sensível e corre risco alto de deteriorar-se, o processo é acelerado e dá início logo após o abate, a deterioração dos músculos resulta na perda da qualidade da carne do pescado (Pacheco-Aguilar, Lugo-Sánchez \& Robles-Burgueño, 2000).

É necessário manusear com cuidado a carne do pescado no processo de produção para não correr risco de contaminação. É indispensável o uso frequente e Boas Práticas de Manipulação, APPCC (Análise de Perigos e Pontos Críticos de Controle) para que haja sempre pescados com qualidade e seguros (Akinyemi Ko et al., 2017).

Sendo responsável pela mudança bioquímica dos músculos, o rigor mortis interfere na qualidade, porém depende da molécula que leva energia (ATP), e até o momento da morte o peixe tem reserva energética (glicogênio). O manuseio do animal tem grande importância para o glicogênio, sem esquecer os cuidados na hora do abate (Robb \& Kestin, 2002). Valor calórico, assim como: aparência, cor, odor e sabor são considerados características sensoriais, pode-se usar a proteína e lipídios do peixe como responsáveis por impulsionar a deterioração, e acompanhar o tempo para putrefação (Robb \& Kestin, 2002).

O processo de deterioração do pescado é avaliado através dos parâmetros físico-químicos, e para avaliar a textura do pescado observa-se a rigidez (rigor) e se tem capacidade de retenção de água. A análise do pH é estudado para avaliar carnes frescas de peixes comprovando informações sobre como o pescado foi conservado, é observado se houve processo de deterioração e decomposição de aminoácidos, pois através dessa análise junto à outros parâmetro observa-se a alteração nos níveis de pH (Chytiri et al., 2004).

Produzidas pelas enzimas endógenas, e responsáveis pela perda da qualidade da carne, com evolução para o estado de putrefação, as bases nitrogenadas voláteis (N-BVT) é o teste que determina o grau de frescor dos peixes (Chytiri et al., 2004). A utilização dos dispositivos analíticos - biossensores, vem sendo estudada para ser utilizada na indústria, precisamente na avaliação da qualidade dos alimentos, em diferentes áreas (Draisci et al., 1998). 


\subsection{Condição de processamento e preservação do produto}

O método de congelamento é amplamente utilizado para conservação de pescado, em ambiente doméstico e comercial. No entanto, mesmo congelado, processos físico-químicos, biológicos e mudanças na estrutura muscular do pescado podem ocorrer em determinado grau, comprometendo a qualidade sensorial e funcional do produto ao longo do tempo. A desnaturação proteica, diminuição da retenção de água, degradação do ATP (majoritariamente causada por bactérias e enzimas) e oxidação lipídica, são exemplos de alterações que ocorrem no pescado congelado (Kaale et al., 2011; LI et al., 2019).

As alterações autolíticas iniciam logo após a morte do pescado, quando cessa o fornecimento de oxigênio resultando na redução do ATP, favorecendo o início da autólise dos tecidos (quebra das proteínas e lipídios) pela ação das enzimas proteolíticas e lipídicas (Abreu, 2014; Vala, 2016). O óxido trimetilamina (OTMA) nos pescados pode ocorrer em diferentes concentrações, sendo reduzido a trimetilaminas (TMA) por ação enzimática. Entretanto, quando congelado, este processo é inibido no pescado (Abreu, 2014).

A degradação dos lipídios pode ser influenciada por diversos fatores, entre eles: a composição dos ácidos graxos, teor e atividade pró e antioxidante, temperatura de armazenamento, pressão, contato com oxigênio, atividade da água e frescor no momento do congelamento. Um problema relacionado ao pescado rico em lipídios armazenado seco ou congelado é a rancificação, que leva a perda de qualidade e diminui a vida útil do produto em função do aparecimento de sabor desagradável (Dehghani et al., 2018). Adicionalmente, ao ocorrer a liberação das enzimas lipase e fosfolipase no processo de congelamento, as mesmas ficam ativas, iniciando a degradação dos substratos que se encontram no meio devido ao rompimento da estrutura celular ocorrida na formação de cristais de gelo (Rocha, 2013).

Souza et al (2020), constatou que a cor do filé é um atributo de qualidade importante na decisão de compra de um produto. Estes autores avaliaram a coloração dos filés congelados de tilápia (Oreochromis niloticus) e panga (Pangasius hypophthalmus) adquiridos em supermercado e, observaram alterações de cor que podem ser ocasionadas por ressecamento ou oxidação, principalmente na linha de sangue, o que pode causar rejeição por parte dos consumidores. Duan et al (2010), avaliando a qualidade do filé de bacalhau (Ophiodon elongates) observaram mudança na cor, mesmo quando os filés foram revestidos com quitosana incorporada com óleo de peixe.

\subsection{Análise sensorial}

A análise sensorial é a chave para o setor alimentício, pois através dessa avaliação crítica é analisada a aceitabilidade do produto para o comércio e a sua qualidade. A análise está fortemente relacionada ao plano de controle de qualidade de uma empresa, e ainda seus resultados são utilizados para determinar o tempo de prateleira do produto (EMBRAPA, 2020).

O conjunto de todos os complexos processos bioquímicos, físicos, químicos e microbiológicos implicam diretamente em alterações sensoriais que vão promover modificação da textura, odor, aparência e sabor durante todo o processo de armazenamento (Gonçalves, 2011). A análise sensorial (utilizando sentidos como visão, tato, paladar, olfato e audição) em conjunto com parâmetros físico-químicos e microbiológicos representam o método mais confiável para aferir o grau de frescor do pescado. Na aparência externa, odor e textura são avaliados conforme às espécies, como parâmetro os peixes que recebem zero pontos são considerados peixes frescos, já os em processos de deterioração com o tempo de armazenamento recebem até 3 pontos (Bogdanović et al., 2012).

O frescor do peixe está diretamente ligado à aceitabilidade, devido ser um alimento com maior probabilidade de deterioração, os consumidores avaliam com maior critério em comparação a outros perecíveis. Sendo fundamental garantir a qualidade do pescado durante todo o processo produtivo para garantir a aceitação (Amaral \& Freitas, 2013). 


\subsection{Comercialização}

Segundo a FAO (2016), pesquisas relatam que há uma estimativa equivalente a 130 milhões de toneladas de peixe da água doce e salgada, entre outros; como crustáceos e moluscos, anualmente são capturados, sendo que equivalente a 7,3 milhões de toneladas são direcionados ao descarte, equivalente a $1 / 4$ do total produzido. Observa-se que o descarte é maior do que o aproveitamento, e a parte descartada polui o meio ambiente (Vidotti \& Gonçalves, 2006).

O reaproveitamento do material residual de pescados traz vantagens econômicas para a indústria agregando valores, além de sanar a eliminação de resíduos orgânicos, poluentes e com descarte difícil. Alguns anos atrás esses tipos de resíduos não tinham nenhum valor comercial (Aguiar \& Goulart, 2014).

A representatividade da Região Norte do país traz a importância recursos pesqueiros. Pode-se afirmar através do alto consumo de peixe pela população ribeirinha, que tem o maior registro segundo pesquisas; assim como o consumo na zona urbana e no setor industrial (Macgrath et al., 1993; Goulding et al., 1996).

Diversos estudos com amplas pesquisas são direcionados para a frota pesqueira comercial, mesmo com a importância da pesca industrial e para frigoríficos, tem poucos estudos relacionados (Almeida \& Almeida, 2016).

Segundo Cabral e Almeida, (2006) a piramutaba (B.Vaillantii) é uma das espécies mais comercializada no mercado internacional, assim como outros peixes-lisos, como o surubim (P.Fasciatum) e o mapará (H.Edentatus), e os crustáceos. O mercado local tem preferência para as espécies de escama como o tambaqui, o tucunaré e o jaraqui, enquanto no mercado nacional destaca-se os bagres.

O mercado Internacional trabalha com pouca diversificação, porém com maior custo, pode-se destacar o camarão, lagosta e peixes em filé congelado e peixe fresco. os peixes sofrem maior processamento no mercado nacional, sendo vendidos peixe fresco eviscerado, camarão sem cabeça e filés de peixe. A comercialização no mercado local é preferencialmente pescados inteiros ou eviscerados congelado (Cabral Jr \& Almeida, 2006).

O pirarucu é muito utilizado na culinária do comércio exterior e no Brasil, principalmente na Região Norte, sendo ele fresco para assados e cozidos, seco ou salgados que após a dessalga pode-se preparar deliciosos pratos, sendo eles: pirarucu de casaca, pirarucu de forno com leite de coco, gratinados, entre outros. A demanda do pirarucu salgado vem aumentando de vido a sua palatabilidade agradável, as fábricas vêm sendo incentivadas para o uso da salga, sendo necessário para o processamento dentro dos padrões de segurança alimentar (Nunes et al., 2012).

Biojóias produzidas de forma artesanal a partir da escama do pirarucu como; bolsas, colares, brincos, pulseiras e anéis, vem ganhando aceitação no mercado local, regional e internacional. Sendo assim, pode-se notar a importância do artesanato e sua qualificação, para a renda e produção sustentável.

\section{Conclusão}

O pirarucu é um pescado de elevado valor cultural e comercial. Porém durante o processamento após o abate o risco de contaminação é alto, devido suas características gerais que contribuem para a modificando a sua qualidade e frescor.

O conhecimento dos fatores envolvidos (físico-químicos, microbiológicos e sensoriais) na progressão da deterioração é fundamental para a segurança alimentar aos consumidores finais e para protocolos de beneficiamento e armazenamento ideal nas indústrias.

Diante disto, nota-se ainda a grande importância que este alimento possui tanto para a população como para o desenvolvimento da região, assim como para todo o comércio no Brasil e exterior devido a reutilização de suas escamas pelos artesãos e seu valor comercial na culinária, é um peixe de grande aceitabilidade. 


\section{Referências}

Abreu, L. M. S. (2014). Avaliação de risco em empresa de processamento de peixe congelado. $106 \mathrm{f}$. Dissertação (mestrado em tecnologia alimentar) - Escola Superior Agrária de Santarém, Santarém.

Almeida, O. T. \& Almeida, B. (2016). Caracterização e análise financeira da indústria pesqueira. A indústria pesqueira da Amazônia. Pró Várzea/IBAMA, 110.

Aguiar, G. P. S. \& Goulard, G. A. S. (2014). Produção de óleo e farinha de peixe a partir de coprodutos de pescado provenientes da Bacia Tocantins-Araguaia. Revista eletrônica da UNIVAR, 1(11), 67-71.

Amaral, G. V. \& Freitas, D. G. C. (2013). Método do índice de qualidade na determinação do frescor do peixe. Ciência Rural., Santa Maria, 43(11), 2093 2100 .

Ackman, R. G. (1989). Nutritional composition of fats in seafoods. Progress in Food and Nutrition Science. USA, 13(3-4), 161-241.

Akinyemi, K., Iwalokun, B.A., Oyefolu, A.O.B. \& Fakorede, C.O. (2017). O occurrence of extended-spectrum and ampc ß-lactamases in multiple drug resistant salmonella isolates from clinical samples in lagos. Infect Drug Resist., Nigeria, 10, 19-25.

Badolato, E. S., Carvalho, J. B., Amaral Mello, M. R. P., Tavares, M., Campos, N. C., Auedpimentel, S. \& Morais, C. (1994). Centesimal composition of fatty acids and caloric-value of five marine fish species in the different seasons. Revista Instituto Adolfo Lutz, 5(1), 27-35.

Baixas, N, S., Bover, C. S., Veciana, N. T., Nunes, M. L. \& Vidal, C. M. C. (2003). Development of a quality index method to evaluate freshness in mediterraneanhake (Merluccius merluccius). Journal of Food Science, Chicago, 68(3), 1067-1071.

Botta, Junior. (1991). Intrument for non-destructive texture measuremenf of raw Atlantic cod (Godus morhua) fillet. J. Food Sci., 56(4), 962-964.

Bogdanović, T., Šimat, V., Frka-Roić, A. \& Marković, K. (2012). Development and Application of Quality Index Method Scheme in a Shelf-Life Study of Wild and Fish Farm Affected Bogue (Boops boops, L.). Journal of Food Science, 77(2), 99-106.

Cabral, J. R. W. \& Almeida, O. T. (2006). Avaliação do mercado da indústria pesqueira na Amazônia. In Almeida,O.T. A indústria pesqueira na Amazônia.1 ed. Manaus: Pró-Várzea/IBAMA, 18-39.

Centeno, L., Silva-Acuña. R., Barrios, R., Lugo, R. S., Matute, C. P. \& José, L. (2007). Hematological characteristics of cachama (Colossoma macropomum) in three phases of the growth in Delta Amacuro, Venezuela. Zootecnia Tropical, 25(4), 237-243.

Chytiri, S., Chouliara, I., Savvaidis, I. N. \& Kontominas, M. G. (2004). Microbiological, chemical and sensory assessment of iced whole and filleted aquacultured rainbow trout. Journal of Food Microbiology, New York, 21(2), 157-165.

Contreras-Guzman, E. C. (1994). Bioquímica de pescado e derivados. Funep., 409.

Córser, P. I., Ferrari, G. T., Martínez, Y. B., Salas, E. M. \& Gagnasso, M. A. (2000). Análisis proximal, perfil de ácidos grasos, aminoácidos esenciales y contenido de minerales en doce espécies de pescados de importância comercial em Venezuela. ALAN, 50(2), $187-194$.

Crescêncio, Roger. (2001). Treinamento alimentar de alevinos de pirarucu, Arapaima gigas (Cuvier, 1829), utilizando atrativos alimentares. 35 f. Dissertação (Mestrado em Biologia de Água Doce e Pesca Interior) - Instituto Nacional de Pesquisas da Amazônia, Fundação Universidade do Amazonas, Manaus.

Dehghani, S., Hosseini, S. V. \& Regenstein, J. M. (2018). Edible films and coatings in seafood preservation: A review. Food Chemistry, $240,05-513$.

Draisci, R., Volpe, G., Lucentini, L., Cecilia, A., Federico, R. \& Palleschi, G. (1998). Determination of biogenic amines with an electrochemical biosensor and its application to salted anchovies. Food Chemistry, 62(2), 225-232.

Duan, J., Cherian, G. \& Zhao, Y. (2010). Quality enhancement in fresh and frozen lingcod (Ophiodon elongates) fillets by employment of fish oil incorporated chitosan coatings. Food Chemistry, 119(2), 524-532.

EMBRAPA - Empresa Brasileira de Pesquisa Agropecuária. (2020). Parque Estação Biológica - PQEB. https://www.embrapa.br/mapa-do-site.

FAO. (2016). The State of World Fisheries and Aquaculture - Contributing to food security and nutrition for all. Rome. 200.

Galvis, G., Mojica, J. I., Duque, S. R., Castellanos, C., Sánchez-Duarte, P., Arce, M., Guitiérrez, A., Jiménez, L. F., Santos, M., Vejarano, S., Arbeláez, F., Prieto, E., Leiva, M. \& Calle, J. C. (2006). Peces del médio Amazonas: Región de Letticia. Conservación Internacional. Serie de Guías Tropicales de Campo n5. Editorial Panamericana, 548.

Gonçalvez, A. A. (2011). Tecnologia do pescado: ciência, tecnologia, inovação e legislação (ed). Editora Atheneu. 608.

Gurgel, J. J. S. \& Freitas, J. V. F. (1972). Sobre a Composição Química de Doze Espécies de Peixe de Valor Comercial de Açudes do Nordeste Brasileiro. Boletim Técnico do DNOCS, 30(1), 45-57.

Goulding, M., Smith, N. J. H. \& Mahar, D. J. (1996). Inundações da fortuna: ecologia e economia na Amazônia. Columbia University Press.

Hylding, G. \& Nilsen, D. (2001). A review of sensory and instrumental methods used to evaluate the texture of fish muscle. Journal of Texture Studies, 32(3), $219-242$.

Huss, H. H. (1998). (Ed.). El Pescado Fresco: su Calidad y Cambios de su Calidad. (Documento Técnico de Pesca, n. 348). Roma: FAO, 202.

Jacquot, T. R. (1989). Organics constituentes of and other aquatic foods. In: Sanchez, L. Pescado, material prima e processamento. Fundação Cargill. Campinas. 
Kaale, L. D., Eikevik, T. M., Rustad, T. \& Kolsaker, K. (2011). Superchilling of food, a review. J Food Eng., 107(2), 141-146.

Kirschnik, P. (2007). Avaliação da estabilidade de produtos obtidos de carne mecanicamente separarda de tilápia nilótica (Oreochromis niloticus). $92 \mathrm{f}$. Tese (Doutorado em Aquicultura) - Centro de Aqüicultura. Universidade Estadual Paulista, Jaboticabal.

Könche, José Carlos. (2011). O fluxograma da pesquisa cientifica In: Könche, José Carlos. Fundamentos de metodologia científica: teoria da ciência e iniciação à pesquisa. Petrópolis, RJ: Vozes, 122.

Landgraf, Mariza. (1996). Deterioração microbiana de alimentos. In: Franco, B. D. G; Landgraf f, M. Microbiologia dos Alimentos. Editora Atheneu, 93-108.

Li, D., Qin, N., Zhang, L., Li, Q., Prinyawiwatkul, W. \& Luo, Y. (2019). Degradation of adenosine triphosphate, water loss and textural changes in frozen common carp (Cyprinus carpio) fillets during storage at different temperatures. International Journal of Refrigeration, 98, $294-301$.

Nunes, E. S. C. L., Franco, R. M., Mársico, E. T. \& Neves, M. S. (2012). Qualidade do pirarucu (Arapaima gigas Shinh, 1822) salgados secos comercializados em mercados varejistas. Instituto Adolfo Lutz. 71(3). 520 -529.

Markore, T., Hansen, A. A., Unander, E. \& Einen, O. (2002). Composition of liquid holding capacity and mechanical properties of farmed raibow trout: variation between fillet selection and the impacto f ice frozen storage. Journal of Food Science, 67, 1933-1938.

Oetterer, M. (2002). Industrialização do pescado cultivado. Agropecuária, 200.

Ozogul, Y. \& Ozogul, F. (2004). Effects of slaughtering methods on sensory, chemical and microbiological quality of rainbow trout (Onchorynchus mykiss) stored in ice and MAP. European Food Research and Technology, 219(3), 211-216.

Pacheco-Aguilar, R., Lugo- Sánchez, M. E. \& Robles-Burgueño, M. R. (2000). Postmortem biochemical and functional characteristics of monterey sardine muscle stored at $0^{\circ} \mathrm{C}$. Journal of Food Science, Chicago, 65(1), 40-47.

Fabré, N. N. \& Barthem, R. B. (2005). O manejo da pesca dos grandes bagres migradores: pitamutaba e dourada no eixo Solimões-Amazonas. Ibama., Manaus.

Robb, D. \& Kestin, S. (2002). Methods used to kill fish: field observations and literature reviewed. Animal Welfare, Hertfordshire, 11(3), 269-282.

Rocha, C. M. C. D., Resende, E. K. D., Routledge, E. A. B. \& Lundsted, L. M. (2013). Avanços na pesquisa e no desenvolvimento da aquicultura brasileira. Pesquisa agropecuária brasileira, Brasilia, 48(8), IV-VI.

Scorvo Filho, J. D. (2004). O agronegócio da aqüicultura: perspectivas e tendências. Zootecnia e o Agronegócio.

Soares, K. M. P. \& Gonçalves, A. A. (2012). Qualidade e segurança do pescado. Rev Inst Adolfo Lutz. São Paulo, 71(1), 1-10.

Souza, A.F.L. (2016). Rendimento, caracterização físico-química e composição em ácidos graxos de peixes siluriformes da Amazônia. Tese de Doutorado em Ciências Pesqueiras nos Trópicos. Universidade Federal do Amazonas - UFAM.

Souza, J. T., Da Silva, É. L. B., Da Silva, L. R. C., De Oliveira, P. V. C., Da Silva Luz, K. S., Firmino, S. S., Da Silva, P. G., Rebouças, L. O. S. \& Lima, P. O. (2020). Comparação físico-química e sensorial de filés congelados de Oreochromis niloticus e Pangassius hypothtalmus. Research, Society and Development, 9(10), 1-17.

Stansby, M. E. \& Olcott, H. E. (1968). Composición de pescado. In: Sanchez, L. Pescado. Matéria-prima e processamento. Fundação Cargill, Campinas.

Tavares-Dias, M., Moraes, F.R. \& Martins, M.L. (2008). Hematological assessment in four Brazilian teleost fish with parasitic infections collected in feefishing from Franca, São Paulo, Brazil. Boletim do Instituto de Pesca, 34, 189-196.

Tavares, D. \& Moraes, F. R. (2004). Hematology of teleosts fish. Villimpress, Ribeirão Preto. 144.

Tavares, D. \& Moraes, F.R. (2007). Haematological and biochemical reference intervals for farmed channel catfish. Journal of Fish Biology, 71, $383-388$.

Tavares-Dias, M., Affonso, E. G., Oliveira, S. R., Marcon, J. L. \& Egami, M. I. (2008). Comparative study on hematological parameters of farmed matrinxã, Brycon amazonicus Spix and Agassiz, 1829 (Characidae: Bryconinae) with others Bryconinae species. Acta Amazonica., Manaus, 38(4), 799-806.

Tavares, M. \& Gonçalves, A. (2011). Aspectos Físico-químicos do Pescado. In: Gonçalves, A. A. (Ed.). Tecnologia do Pescado. Atheneu. 10-20.

Torres, E. A. F. S., Campos, N. C., Duarte, M., Garbelotti, M. L., Philippi, S. T. \& Rodrigues, R. S. M. (2000). Composição centesimal e valor calórico de alimentos de origem animal. Ciênc. Tecnol. Aliment. 20(2), 145-50.

TACO - Tabela Brasileira De Composição De Alimentos. (2011). (4a ed.), NEPA - UNICAMP, 161.

UNESP - Faculdade De Ciências Agronômicas. (2015). Tipos de revisão de literatura. https://www.fca.unesp.br/Home/Biblioteca/tipos-de-evisao-deliteratura.pdf.

Vala, M. O. (2016). Aplicação de revestimentos edíveis à base de subprodutos da indústria do pescado na preservação de atum fresco. $92 \mathrm{f}$. Tese de mestrado em gestão da qualidade e segurança alimentar. Escola superior de Turismo e Tecnologia do Mar - Peniche.

Venturieri, R. \& Bernardino, G. (1999). Pirarucu espécie ameaçada pode ser salva através do cultivo. Panorama da Aquicultura., 13-21.

Vidotti, R. M. \& Gonçalves, G. S. (2006). Produção e caracterização de silagem, farinha e óleo de tilápia e sua utilização na alimentação animal. Pesquisador Científico - Centro Avançado de Pesquisa Tecnológica do Agronegócio do Pescado Continental Instituto de Pesca - APTA - SAA. 